\title{
ABOUT THE DECAY OF SURFACE WAVES ON VISCOUS FLUIDS WITHOUT SURFACE TENSION
}

\author{
GERHARD STRÖHMER \\ Department of Mathematics, University of Iowa \\ Iowa City, IA, 52242, USA \\ E-mail: strohmer@math.uiowa.edu
}

\begin{abstract}
We study the decay of the motions of a viscous fluid subject to gravity without surface tension with a free boundary at the top. We show that the solutions of the linearization about the equilibrium state decay, but not exponentially in a uniform manner. We also discuss the consequences of this for the non-linear equations.
\end{abstract}

1. Introduction. For systems of parabolic equations for functions defined on a bounded domain it seems plausible for reasons of compactness of the operators involved that if all solutions converge to an equilibrium state, then this convergence should occur at an exponential rate. This is indeed even true for some examples of compressible viscous fluid flow without surface tension on the boundary, although the system of equations in question is not really parabolic. Zajacczkowski [12] showed that for such a fluid not exposed to an exterior force, but with a positive external pressure, and with small initial values all solutions converge exponentially in a uniform way to an equilibrium solution. We show that this is no longer true as soon as the fluid is exposed to the force of gravity.

This paper consists of two unequal parts. The first one is rigorous and shows that the solutions of the linearization of the equation around the equilibrium do not decay exponentially in a uniform fashion. It consists of Sections 2 to 4 . Then, in a less rigorous form, in Sections 5 and 6, we compute the linearization of the equations and discuss the consequences of the linear result for the actual non-linear equations for the fluid. These considerations could also be made more rigorous, but that would go beyond the scope of the present paper.

Now we describe the precise form of our mathematical problem. We consider the flow

The work of the author was in part supported by a grant from the Polish Committee of Scientific Research, number 2 PO3A 02816.

2000 Mathematics Subject Classification: 35Q30, 35M10, 76D05, 16N10.

The paper is in final form and no version of it will be published elsewhere. 
of a barotropic or incompressible viscous fluid without surface tension in a variable region

$$
\Omega_{t}=\left\{\left(x_{1}, x_{2}, x_{3}\right):-h \leq x_{3} \leq f\left(x_{1}, x_{2}, t\right)\right\}
$$

in 3-space with some fixed $h>0$. Both $f$ and the functions introduced later are assumed to be periodic with period $l_{k}$ in the $x_{k}$ directions $(k=1,2)$. Then we can obtain these functions from their values on the basic cell

$$
\Omega_{t}^{0}=\left\{\left(x_{1}, x_{2}, x_{3}\right): 0 \leq x_{1} \leq l_{1}, 0 \leq x_{2} \leq l_{2},-h \leq x_{3} \leq f\left(x_{1}, x_{2}, t\right)\right\} .
$$

It is clear that although the region $\Omega_{t}$ we consider is not bounded, the periodicity assures that all function spaces on this domain have all the compactness properties such spaces for bounded domains usually have. We can alternatively also see this as a problem on the compact manifold we obtain by gluing opposite ends of the intervals $\left[0, l_{k}\right]$ in $x_{1}$ and $x_{2}$ direction together. With a little bit more effort we could carry out our considerations on a ball as well, but we want to spare ourselves the unpleasant aspects of spherical coordinates. Let us denote the regions obtained by setting $f=0$ as $\mathcal{R}$ and $\mathcal{R}^{0}$ respectively. As we want to describe barotropic and incompressible fluids at once we use the fluid velocity $v(x, t)$ and the fluid pressure $p(x, t)$ as the relevant variables. Then the inverse $G:(0,+\infty) \rightarrow(0,+\infty)$ of the equation of state gives us the density $\rho$ as $\rho=G(p)$. We will assume that $G$ is twice continuously differentiable on $(0,+\infty)$, that $G^{\prime}(p) \geq 0$ and that either $G$ is constant or $G^{\prime}(p)>0$ throughout, and also that $G^{\prime}(p)$ is bounded at infinity, but not usually at zero. The stress tensor $\mathbb{T}$ in the fluid is given by

$$
\mathbb{T}(v, p)=\left[\mathbb{T}_{i j}(v, p)\right]=\left[-p \delta_{i j}+\mathbb{D}_{i j}(v)\right]
$$

with

$$
\mathbb{D}(v)=\left[\mathbb{D}_{i j}(v)\right]=\left[\nu_{1}\left(\frac{\partial v_{i}}{\partial x_{j}}+\frac{\partial v_{j}}{\partial x_{i}}\right)+\left(\nu_{2}-\nu_{1}\right) \delta_{i j} \operatorname{div}(v)\right],
$$

where $\nu_{1}$ and $\nu_{2}$ are the viscosity coefficients. Also let $\mathbf{e}_{3}=(0,0,1)$. Then the equations

$$
\begin{aligned}
\rho\left(v_{t}+v \cdot \nabla v\right)-\operatorname{div}(\mathbb{T}(v, p)) & =-g \rho \mathbf{e}_{3} \\
\rho_{t}+\operatorname{div}(\rho v) & =0
\end{aligned}
$$

are fulfilled in the interior of the fluid. ( $g$ is the acceleration due to gravity.) We assume that the pressure at the top side equals $\mathrm{p}_{0}>0$, and the velocity equals zero at the bottom. Let $\mathbf{n}$ be the outward normal to the top part of $\partial \Omega_{t}$. Then, as we leave the surface tension out of consideration, our boundary conditions are

$$
\begin{aligned}
\mathbb{T}(v, p) \cdot \mathbf{n} \mid\left\{x_{3}=f\left(x_{1}, x_{2}, t\right)\right\} & =-\mathrm{p}_{0} \mathbf{n} \\
v \mid\left\{x_{3}=-h\right\} & =0 .
\end{aligned}
$$

We assume that $\nu_{1}$ and $\nu_{2}$ fulfil the condition $\nu_{2}>\nu_{1} / 3>0$. This inequality implies that $\mathbb{D}_{i j}(v) v_{i x_{j}}$ is a positive semidefinite quadratic form in $\nabla v$ which is zero only if $\mathbb{D}_{i j}(v)=0$. We obtain another equation, which is due to the fact that all fluid particles at the position $x(t)=\left(x_{1}(t), x_{2}(t), x_{3}(t)\right)$ are moving at the speed $\left(v_{1}(x(t), t), v_{2}(x(t), t), v_{3}(x(t), t)\right)$ and particles once on the boundary always stay there. So by differentiating the equation $x_{3}(t)=f\left(x_{1}(t), x_{2}(t), t\right)$ with respect to time we obtain

$$
v_{3}\left(x_{1}, x_{2}, x_{3}, t\right)=\sum_{k=1}^{2} f_{x_{k}}\left(x_{1}, x_{2}, t\right) v_{k}\left(x_{1}, x_{2}, x_{3}, t\right)+f_{t}\left(x_{1}, x_{2}, t\right),
$$


if $x_{3}=f\left(x_{1}, x_{2}, t\right)$.

Note that if $G$ is constant, then the equation $\rho_{t}+\operatorname{div}(\rho v)=0$ becomes $\operatorname{div}(v)=0$. Also note $-g \rho \mathbf{e}_{3}=-\rho \nabla\left(g x_{3}\right)$, so by defining $U=-g x_{3}$ we have $-g \rho \mathbf{e}_{3}=\rho \nabla U$.

If $\left(v_{e}, p_{e}\right)$ on a constant domain $\Omega_{e}$ is a time-independent solution of (1) with $v_{e}=0$, then (1) becomes

$$
\nabla p_{e}=-G\left(p_{e}\right) g \mathbf{e}_{3}
$$

This means that $p_{e}$ only depends on $x_{3}$ and so with the boundary condition

$$
p_{e}\left(x_{1}, x_{2}, f\left(x_{1}, x_{2}, t\right), t\right)=\mathrm{p}_{0}
$$

we get $f$ is constant. This allows us to compute the equilibrium solution for compressible and incompressible fluids as the solution of an ordinary differential equation, but we will not do that. It is easy to see our conditions imply that the solution is three times differentiable, that we can find such a solution for any given mass, and that we can choose $h$ in relation to the quantity of the fluid in such a way that for the equilibrium state we have $\Omega_{e}=\mathcal{R}$.

As we demonstrate in Section 5, the linearization of these equations about the equilibrium solutions is

$$
\begin{gathered}
\rho_{e} u_{t}-\operatorname{div}(\mathbb{D}(u))+\nabla \alpha=-g G^{\prime}\left(p_{e}\right) \alpha \mathbf{e}_{3}, \\
G^{\prime}\left(p_{e}\right) \alpha_{t}+\operatorname{div}\left(\rho_{e} u\right)=0, \\
\varphi_{t}=u_{3}
\end{gathered}
$$

with the boundary conditions

$$
\mathbb{T}(u, \alpha) \cdot \mathbf{e}_{3}=\left(p_{e}\right)_{x_{3}}(0) \varphi \mathbf{e}_{3} \text { at } x_{3}=0, \text { and } u\left(x_{1}, x_{2},-h, t\right)=0 .
$$

Here $u$ is the "linear equivalent" of $v, \alpha$ that of $p$, and $\varphi$ that of $f$. The "linear equivalent" of the law of the conservation of mass is that

$$
\mathcal{C}(t)=\int_{\mathcal{R}^{0}} G^{\prime}\left(p_{e}\right) \alpha d x+\rho_{e}(0) \int_{\mathcal{R}^{0} \cap\left\{x_{3}=0\right\}} \varphi d \sigma
$$

is constant. Note that $\mathcal{R}^{0} \cap\left\{x_{3}=0\right\} \subset \partial \mathcal{R}^{0}$.

We prove the following two theorems. The first does not contain anything new, but is included to make this argument more self-contained and more easily readable, the second one really states the lack of decay for the linearized equation. They are proved in Sections 3 and 4. The less rigorous considerations are contained in Sections 5 and 6 and their results are not stated here. For the notation used see Section 1.1. Before stating our theorems we define

$$
B_{1}=\left\{u \in L_{2}: \int_{\mathcal{R}^{0}} u \cdot \nabla \Phi d x=0 \quad\left(\Phi \in H^{1} \text { with } \Phi=0 \text { at } x_{3}=0\right)\right\} .
$$

THEOREM 1. For compressible fluids, given $\alpha_{0} \in H^{1}, u_{0} \in L^{2}, \varphi_{0} \in H_{\partial}^{1 / 2}$, there are functions

$$
\begin{gathered}
\alpha \in C^{0}\left([0,+\infty), H^{1}\right) \cap C^{1}\left((0,+\infty), H^{1}\right), \\
v \in C^{0}\left([0,+\infty), L^{2}\right) \cap C^{1}\left((0,+\infty), H^{2}\right), \\
\varphi \in C^{0}\left([0,+\infty), H_{\partial}^{1 / 2}\right) \cap C^{1}\left((0,+\infty), H_{\partial}^{1 / 2}\right),
\end{gathered}
$$


which solve (5) and (6) for $t>0$, while $(u, \alpha, \varphi)(0)=\left(u_{0}, \alpha_{0}, \varphi_{0}\right)$.

For incompressible fluids, given $u_{0} \in B_{1}, \varphi_{0} \in H_{\partial}^{1 / 2}$, there are functions

$$
\begin{gathered}
\alpha \in C^{1}\left((0,+\infty), H^{1}\right), \quad v \in C^{0}\left([0,+\infty), L^{2}\right) \cap C^{1}\left((0,+\infty), H^{2}\right), \\
\varphi \in C^{0}\left([0,+\infty), H_{\partial}^{1 / 2}\right) \cap C^{1}\left((0,+\infty), H_{\partial}^{1 / 2}\right),
\end{gathered}
$$

which solve (5) and (6) for $t>0$, while $(u, \varphi)(0)=\left(u_{0}, \varphi_{0}\right)$.

In many of our statements we use the function $G^{\prime}\left(p_{e}\right)$, which equals zero for incompressible flows and is bounded from above and away from zero for compressible flows to make a unified statement for both cases.

THEOREM 2. If $\mathcal{C}(0)=0$ for the solutions of Theorem 1 , then as $t \rightarrow \infty$ the functions $u(t), G^{\prime}\left(p_{e}\right) \alpha(t)$ converge to zero at least weakly in $L^{2}$, and $\varphi(t)$ weakly in $L_{\partial}^{2}$, but for no $s \geq 0, \beta_{1} \in[s, s+1 / 2], \beta_{2} \in[s, s+3 / 2]$ are there numbers $\gamma>0, C<\infty$, such that

$$
\begin{aligned}
\|u(t)\|_{H^{\beta_{2}}}+\left\|G^{\prime}\left(p_{e}\right) \alpha(t)\right\|_{H^{\beta_{1}}} & +\|\varphi(t)\|_{H_{\partial}^{s}} \\
& \leq C e^{-\gamma t}\left(\|u(0)\|_{H^{\beta_{2}}}+\left\|G^{\prime}\left(p_{e}\right) \alpha(0)\right\|_{H^{\beta_{1}}}+\|\varphi(0)\|_{H_{\partial}^{s}}\right)
\end{aligned}
$$

for all such functions and all $t>0$.

The convergence results could be improved, but we do not want to do this here. In the last two sections we indicate how this translates into a lack of exponential decay for our non-linear equation.

It should be pointed out that in [3] a problem similar to the nonlinear incompressible problem is considered, where the periodicity in $x_{1}, x_{2}$ direction is replaced by the condition that the perturbations go to zero at infinity in space. Beale shows that at least if one assumes twice differentiable dependence of the solution on the initial value over the entire infinite interval, solutions cannot always exist.

I am indebted to W. Zajaczkowski from the Institute of Mathematics of the Polish Academy of the Sciences for many crucial discussions.

1.1. Notation. We denote generic constants by $C$. The norm of any Banach space $\mathcal{B}$ is denoted by $\|\cdot\|_{\mathcal{B}}$. Let $S \subset \mathbb{R}^{m}$ be an open set or its closure. Then $C^{k+\alpha}(S, \mathcal{B})$ for $(k=0,1,2, \ldots, \alpha \in[0,1])$ are the spaces of all functions with values in $V$ that are, locally in $S$, Hölder-continuous with exponent $\alpha$, the same being true for the derivatives up to order $k$. This means that these are Banach spaces with the usual norms only in the case that $S$ is compact. By $C_{0}^{k+\alpha}(S, \mathcal{B})$ we denote the space of functions in $C^{k+\alpha}(S, \mathcal{B})$ with compact support in $S$.

Then if $\Omega$ is open, $L^{p}(\Omega, \mathcal{B})$ is the closure of $C_{0}^{0}(\Omega, \mathcal{B})$ with respect to the norm

$$
\left(\int_{\Omega}\|u(x)\|_{\mathcal{B}}^{p} d x\right)^{1 / p}
$$

and $L_{\text {loc }}^{p}(\Omega, \mathcal{B})$ consists again of the functions that locally belong to $L^{p}(\Omega, \mathcal{B}) . L^{\infty}(\Omega, \mathcal{B})$ is the set of all elements of $L_{\text {loc }}^{1}(\Omega, \mathcal{B})$ that are essentially bounded in addition. If $\mathcal{B}=\mathbb{R}^{n}$ or $\mathcal{B}=\mathbb{C}^{n}$ we will leave $\mathcal{B}$ out in the notation from now on, unless confusion can arise from this. For any positive integer $k$ we denote by $W_{p}^{k}(\Omega)$ the set of all functions that have distributional derivatives up to $k$-th order that lie in $L^{p}(\Omega)$. For other $\mu \geq 0$ we 
define $W_{p}^{\mu}(\Omega)$ by appropriate real interpolation (see [10]), and $W_{p 0}^{\mu}(\Omega)$ is the closure of the set of infinitely often differentiable functions with compact support in $W_{p}^{\mu}(\Omega)$.

For any of the Sobolev spaces $W_{p}^{\mu}(\Omega)$, if $p=2$ we will always omit the subscript, e.g. $W^{\mu}(\Omega)=W_{2}^{\mu}(\Omega)$. In addition we define

$$
\begin{aligned}
W_{p}^{\mu}=\left\{u \mid\left(0, l_{1}\right) \times\left(0, l_{2}\right) \times(-h, 0): u\right. & \in W_{p}^{\mu}\left(\left(0,2 l_{1}\right) \times\left(0,2 l_{2}\right) \times(-h, 0)\right), \\
u\left(x+l_{1} \mathbf{e}_{1}\right) & \left.=u(x), u\left(x+l_{2} \mathbf{e}_{2}\right)=u(x)\right\}, W^{\mu}=W_{2}^{\mu} .
\end{aligned}
$$

For function spaces on the surface $\left\{x_{3}=0\right\} \cap \mathcal{R}^{0}$ we use the notation $W_{p \partial}^{\mu}, W_{\partial}^{\mu}$ or $L_{\partial}^{p}$ in an analogous way. We also use the summation convention that if an index occurs in more that one place in an expression this actually stands for the sum of these expressions in which this index runs through its natural range.

2. Some preliminary considerations. In Sections 2 and 3 we allow complexvalued functions for technical reasons. Let us first consider the following two systems of equations, which are obviously related to the linearized system. They are

$$
\begin{gathered}
-\operatorname{div}(\mathbb{D}(u))+\nabla \alpha+g G^{\prime}\left(p_{e}\right) \alpha \mathbf{e}_{3}=f_{1}, \\
\operatorname{div}\left(\rho_{e} u\right)=f_{2}, \\
\mathbb{T}(u, \alpha) \cdot \mathbf{e}_{3}=H \text { at } x_{3}=0, u=0 \text { at } x_{3}=-h,
\end{gathered}
$$

and

$$
\begin{gathered}
-\operatorname{div}(\mathbb{D}(u))=f, \\
\mathbb{D}(u) \cdot \mathbf{e}_{3}=H \text { at } x_{3}=0, \quad u=0 \text { at } x_{3}=-h .
\end{gathered}
$$

We have

Lemma 3. Assume all the variables below fulfil the system of equations (8). Then

$$
\int_{\mathcal{R}^{0}} \mathbb{D}_{i j}(u) \bar{u}_{i x_{j}} d x=\int_{\mathcal{R}^{0} \cap\left\{x_{3}=0\right\}} H \cdot \bar{u} d \sigma+\int_{\mathcal{R}^{0}} f_{1} \bar{u}+\rho_{e}^{-1} \overline{f_{2}} \alpha d x .
$$

If they fulfil (9) then

$$
\int_{\mathcal{R}^{0}} \mathbb{D}_{i j}(u) \bar{u}_{i x_{j}} d x=\int_{\mathcal{R}^{0} \cap\left\{x_{3}=0\right\}} H \cdot \bar{u} d \sigma+\int_{\mathcal{R}^{0}} f \bar{u} d x .
$$

Proof. Let us first prove the second claim. Multiplying the first equation in (9) by $\bar{u}$ we have

Integrating by parts we get

$$
-\int_{\mathcal{R}^{0}} \operatorname{div}(\mathbb{D}(u)) \cdot \bar{u} d x=\int_{\mathcal{R}^{0}} f \cdot \bar{u} d x .
$$

$$
\int_{\mathcal{R}^{0}} \mathbb{D}_{i j}(u) \cdot \bar{u}_{i x_{j}} d x-\int_{\partial \mathcal{R}^{0}} \mathbf{n} \cdot \mathbb{D}(u) \cdot \bar{u} d \sigma=\int_{\mathcal{R}^{0}} f \cdot \bar{u} d x .
$$

Now the integrals over all boundaries except the top boundary drop out and there we have $\mathbf{n} \cdot \mathbb{D}(u) \cdot \bar{u}=H \cdot \bar{u}$, so

$$
-\int_{\mathcal{R}^{0} \cap\left\{x_{3}=0\right\}} H \cdot \bar{u} d \sigma+\int_{\mathcal{R}^{0}} \mathbb{D}_{i j}(u) \bar{u}_{i x_{j}} d x=\int_{\mathcal{R}^{0}} f \cdot \bar{u} d x .
$$

This proves our second claim. 
For the first one notice that any solution of (8) also fulfils (9) with $H$ replaced by $H+\alpha \mathbf{e}_{3}$ and $f$ replaced by $f_{1}-\nabla \alpha-g G^{\prime}\left(\rho_{e}\right) \alpha \mathbf{e}_{3}$. Then we have for such solutions

$$
\int_{\mathcal{R}^{0}} \mathbb{D}_{i j}(u) \bar{u}_{i x_{j}} d x=\int_{\mathcal{R}^{0}}\left(f_{1}-\nabla \alpha-g G^{\prime}\left(\rho_{e}\right) \alpha \mathbf{e}_{3}\right) \cdot \bar{u} d x+\int_{\mathcal{R}^{0} \cap\left\{x_{3}=0\right\}} H \cdot \bar{u}+\alpha \bar{u}_{3} d \sigma .
$$

Now

$$
\int_{\mathcal{R}^{0}}-\nabla \alpha \cdot \bar{u} d x=\int_{\mathcal{R}^{0}} \alpha \operatorname{div}(\bar{u}) d x-\int_{\mathcal{R}^{0} \cap\left\{x_{3}=0\right\}} \alpha \bar{u}_{3} d \sigma,
$$

as again all other boundary integrals vanish, and so

$$
\int_{\mathcal{R}^{0}} \mathbb{D}_{i j}(u) \bar{u}_{i x_{j}} d x=\int_{\mathcal{R}^{0}}\left(f_{1}-g G^{\prime}\left(\rho_{e}\right) \alpha \mathbf{e}_{3}\right) \cdot \bar{u}+\alpha \operatorname{div}(\bar{u}) d x+\int_{\mathcal{R}^{0} \cap\left\{x_{3}=0\right\}} H \cdot \bar{u} d \sigma .
$$

Also we can now solve $\operatorname{div}\left(\rho_{e} u\right)=f_{2}$ for $\operatorname{div}(u)$ to obtain

$$
\int_{\mathcal{R}^{0}} \alpha\left(\operatorname{div}(\bar{u})-G^{\prime}\left(p_{e}\right) g \bar{u}_{3}\right) d x=\int_{\mathcal{R}^{0}} \rho_{e}^{-1} \bar{f}_{2} \alpha-\alpha \bar{u}_{3}\left(\rho_{e}^{-1} \rho_{e x_{3}}+G^{\prime}\left(p_{e}\right) g\right) d x .
$$

As due to (4)

$$
\rho_{e}^{-1} \rho_{e x_{3}}+G^{\prime}\left(p_{e}\right) g=\rho_{e}^{-1} G^{\prime}\left(p_{e}\right) p_{e x_{3}}+G^{\prime}\left(p_{e}\right) g=\rho_{e}^{-1} G^{\prime}\left(p_{e}\right)\left(p_{e x_{3}}+\rho_{e} g\right)=0,
$$

our claim is now obvious.

Lemma 4. The system (8) can be solved uniquely for all $H \in H_{\partial}^{1 / 2}, f_{1} \in L^{2}, f_{2} \in H^{1}$, and

$$
\|u\|_{H^{2}}+\|\alpha\|_{H^{1}} \leq C\left(\left\|f_{1}\right\|_{L^{2}}+\left\|f_{2}\right\|_{H^{1}}+\|H\|_{H_{\partial}^{1 / 2}}\right)
$$

If $f_{1}=0$ and $f_{2}=0$ then also

$$
\|u\|_{H^{\mu+3 / 2}}+\|\alpha\|_{H^{\mu+1 / 2}} \leq C\|H\|_{H_{\partial}^{\mu}}
$$

for all $\mu \geq 0$.

Proof. First we prove the solvability and the first estimate. By Lemma 3 with $f_{1}=0$, $f_{2}=0, H=0$ we get that $\mathbb{D}(u)=0$ for such a solution, which implies $u=0$ using the boundary condition at the bottom. Then also $\alpha=0$ at the top due the boundary condition there, and $\nabla \alpha+g G^{\prime}\left(p_{e}\right) \alpha \mathbf{e}_{3}=0$, which implies $\alpha$ only depends on $x_{3}$, and is zero everywhere because it is zero at $x_{3}=0$. So we get the uniqueness of the solution of this equation. As this is an elliptic system in the sense of [2] and the boundary conditions are complementing (see, e.g., [8]), we get the a priori estimate

$$
\|u\|_{H^{2}}+\|\alpha\|_{H^{1}} \leq C\left(\left\|f_{1}\right\|_{L^{2}}+\left\|f_{2}\right\|_{H^{1}}+\|H\|_{H^{1 / 2}}\right) .
$$

This allows us to use the continuity method to conclude that the equation is solvable for all $f_{1}, f_{2}, H$ from the spaces indicated above if this is true for one choice of $G, \nu_{1}, \nu_{2}$ permitted here, e.g. $G=1, \nu_{1}=1, \nu_{2}=1$. Then we must solve

$$
\begin{gathered}
-\operatorname{div}(\mathbb{D} u)+\nabla \alpha=f_{1}, \\
\quad a \alpha+\operatorname{div}(u)=f_{2}, \\
-\mathbb{D}_{j 3}(u)+\delta_{j 3} \alpha=H_{j} \text { at } x_{3}=0, \quad u=0 \text { at } x_{3}=-h
\end{gathered}
$$


for $a=0$, which is again equivalent to solving it for very small $a$. For $a \neq 0$ we can solve for $\alpha$ to obtain $\alpha=a^{-1}\left(f_{2}-\operatorname{div}(u)\right)$. So then the system is equivalent to

$$
-\operatorname{div}(\mathbb{D} u)-a^{-1} \nabla \operatorname{div}(u)=f_{1}-a^{-1} \nabla f_{2}
$$

with the boundary condition

$$
-\mathbb{D}_{j 3}(u)-a^{-1} \delta_{j 3} \operatorname{div}(u)=\widetilde{H}_{j}=H_{j}-a^{-1} \delta_{j 3} f_{2} \text { at } x_{3}=0, \quad u=0 \text { at } x_{3}=-h .
$$

Now for $a>0$ according to [5], Lemma 3.3, this can be solved for all $f_{1}, f_{2}$ and $\widetilde{H}=0$, and so one only needs to find functions with the right boundary values which do not need to solve the equation. It is easy to see one can find such functions of the form $u=x_{3} w\left(x_{1}, x_{2}\right)$, where $w$ is a vector field on $x_{3}=0$. Then at $x_{3}=0$ we have

$$
\mathbb{D}_{j 3}(u)+a^{-1} \delta_{j 3} \operatorname{div}(u)=w_{j}\left(1+\delta_{j 3}\left(1+a^{-1}\right)\right),
$$

and so functions with arbitrary boundary values exist.

Now the second part is clear from [2] if $\mu-1 / 2$ is a non-negative integer, and can be extended by interpolation theory (see [10]) to all $\mu \geq 1 / 2$.

To treat the case $\mu<1 / 2$ let $\Delta_{2}$ be the Laplacian only in the $x_{1}, x_{2}$ directions. Then let $\widehat{u}, \widehat{\alpha}$ be the solution of our problem for the boundary condition $\Delta_{2}^{-1} H$ with $f_{1}=0$, $f_{2}=0$. As $\Delta_{2}$ and the operator we consider here commute, $\Delta_{2} \widehat{u}, \Delta_{2} \widehat{\alpha}$ solve our original problem and

$$
\begin{aligned}
\left\|\Delta_{2} \widehat{u}\right\|_{W^{\mu+3 / 2}}+\left\|\Delta_{2} \widehat{\alpha}\right\|_{W^{\mu+1 / 2}} \leq\|\widehat{u}\|_{W^{\mu+7 / 2}}+\|\widehat{\alpha}\|_{W^{\mu+5 / 2}} & \\
\leq C\left\|\Delta_{2}^{-1} H\right\|_{W_{\partial}^{\mu+2}} \leq C\|H\|_{W_{\partial}^{\mu}} &
\end{aligned}
$$

which proves our claim in general.

3. Analytic semigroups. We want to show that our time evolution equations can be solved by an analytic semigroup. To this end we first consider the system of equations

$$
\begin{gathered}
z \rho_{e} u-\operatorname{div}(\mathbb{D}(u))+\nabla \alpha+g G^{\prime}\left(p_{e}\right) \alpha \mathbf{e}_{3}=\widehat{f}_{1}, \\
z G^{\prime}\left(p_{e}\right) \alpha+\operatorname{div}\left(\rho_{e} u\right)=G^{\prime}\left(p_{e}\right) \widehat{f}_{2}, \\
\mathbb{T}(u, \alpha) \cdot \mathbf{e}_{3}=H \text { at } x_{3}=0, \quad u=0 \text { at } x_{3}=-h
\end{gathered}
$$

for $\operatorname{Re}(z) \geq 0$, and we obtain the following result.

Lemma 5. For $\widehat{f}_{1} \in L^{2}, \widehat{f}_{2}, \alpha \in H^{1}, H \in H_{\partial}^{1 / 2}, u \in H^{2}$ fulfilling the equation (11) we have, if $z$ is sufficiently large,

$$
|z|\|u\|_{L^{2}}+\|u\|_{H^{2}} \leq C\left[\left\|\widehat{f}_{1}\right\|_{L^{2}}+\left\|G^{\prime}\left(p_{e}\right) \widehat{f}_{2}\right\|_{H^{1}}\right]+|z|^{1 / 2}\|H\|_{H_{\partial}^{1 / 2}} .
$$

Proof. Let us first consider the incompressible case. Then $G^{\prime}=0$ and $\rho_{e}=$ const. Then we can use Lemma 3 with $f_{1}=\widehat{f}_{1}-z \rho_{e} u$, and $f_{2}=0$, which gives us the result

$$
z \int_{\mathcal{R}^{0}} \rho_{e}|u|^{2} d x+\int_{\mathcal{R}^{0}} \mathbb{D}_{i j}(u) \bar{u}_{i x_{j}} d x=\int_{\mathcal{R}^{0} \cap\left\{x_{3}=0\right\}} H \cdot \bar{u} d \sigma+\int_{\mathcal{R}^{0}} \widehat{f_{1}} \bar{u} d x,
$$

so also

$$
|z| \int_{\mathcal{R}^{0}} \rho_{e}|u|^{2} d x \leq C\left[\|u\|_{L_{\partial}^{2}}\|H\|_{L_{\partial}^{2}}+\left\|\widehat{f}_{1}\right\|_{L^{2}}\|u\|_{L^{2}}\right]
$$


and

$$
\begin{aligned}
|z|^{1 / 2}\|u\|_{L^{2}} \leq C\left[\|u\|_{L_{\partial}^{2}}^{1 / 2}\|H\|_{L_{\partial}^{2}}^{1 / 2}+\left\|\widehat{f}_{1}\right\|_{L^{2}}^{1 / 2}\|u\|_{L^{2}}^{1 / 2}\right] & \\
& \leq C\|u\|_{L_{\partial}^{2}}^{1 / 2}\|H\|_{L_{\partial}^{2}}^{1 / 2}+C|z|^{-1 / 2}\left\|\widehat{f}_{1}\right\|_{L^{2}}+\frac{1}{2}|z|^{1 / 2}\|u\|_{L^{2}} .
\end{aligned}
$$

Subtracting $\frac{1}{2}|z|^{1 / 2}\|u\|_{L^{2}}$ and multiplying by $|z|^{1 / 2}$ we get

$$
|z|\|u\|_{L^{2}} \leq C\left\|\widehat{f}_{1}\right\|_{L^{2}}+C|z|^{1 / 2}\|u\|_{L_{\partial}^{2}}^{1 / 2}\|H\|_{L_{\partial}^{2}}^{1 / 2} .
$$

Using our equation and the estimate (10) we also get

$$
\|u\|_{H^{2}} \leq C\left[\left\|\widehat{f}_{1}\right\|_{L^{2}}+\|H\|_{H_{\partial}^{1 / 2}}+|z|^{1 / 2}\|u\|_{L_{\partial}^{2}}^{1 / 2}\|H\|_{L_{\partial}^{2}}^{1 / 2}\right] .
$$

By means of the interpolation inequality 10.1 in [4] and the trace estimate

$$
\|u\|_{L_{\partial}^{2}} \leq C\|u\|_{H^{1}}
$$

we obtain that

$$
|z|^{1 / 2}\|u\|_{H^{1}} \leq C\left[\left\|\widehat{f}_{1}\right\|_{L^{2}}+\|H\|_{H_{\partial}^{1 / 2}}\right]+C|z|^{1 / 2}\|H\|_{L_{\partial}^{2}}^{1 / 2}\|u\|_{H^{1}}^{1 / 2} .
$$

Thus, again estimating the last product by a sum of suitable squares and subtracting,

$$
|z|^{1 / 2}\|u\|_{H^{1}} \leq C\left(\left\|\widehat{f}_{1}\right\|_{L^{2}}+\|H\|_{H_{\partial}^{1 / 2}}\right)+C|z|^{1 / 2}\|H\|_{L_{\partial}^{2}} .
$$

Now (12) also implies

$$
|z|\|u\|_{L^{2}} \leq C\left\|\widehat{f}_{1}\right\|_{L^{2}}+C|z|^{1 / 2}\left(\|u\|_{H^{1}}+\|H\|_{L_{\partial}^{2}}\right),
$$

and, estimating $\|u\|_{H^{1}}$ with the previous inequality we get

$$
|z|\|u\|_{L^{2}} \leq C\left\|\widehat{f}_{1}\right\|_{L^{2}}+C\left(\left\|\widehat{f}_{1}\right\|_{L^{2}}+\|H\|_{H_{\partial}^{1 / 2}}+|z|^{1 / 2}\|H\|_{L_{\partial}^{2}}\right),
$$

and this directly implies our claim in this case.

Now let us address the compressible fluid problem. To that end we first consider the system of equations

$$
z \rho_{e} u-\operatorname{div}(\mathbb{D} u)=f
$$

with the boundary condition $u=0$ at the bottom and $\mathbb{D} u \cdot \mathbf{e}_{3}=H$ at the top. Then from the second equation in Lemma 3 we can conclude

$$
|z| \int_{\mathcal{R}^{0}} \rho_{e}|u|^{2} d x \leq C\left[\|u\|_{L_{\partial}^{2}}\|H\|_{L_{\partial}^{2}}+\|f\|_{L^{2}}\|u\|_{L^{2}}\right]
$$

so we have

$$
|z|\|u\|_{L^{2}}^{2} \leq C\left[\|u\|_{L_{\partial}^{2}}\|H\|_{L_{\partial}^{2}}+|z|^{-1}\|f\|_{L^{2}}^{2}\right]+\frac{1}{2}|z|\|u\|_{L^{2}}^{2}
$$

and

$$
|z|\|u\|_{L^{2}} \leq C|z|^{1 / 2}\|u\|_{L_{\partial}^{2}}^{1 / 2}\|H\|_{L_{\partial}^{2}}^{1 / 2}+C\|f\|_{L^{2}} .
$$

As the boundary conditions for the equation are complementing (see [8]) we also get

$$
\|u\|_{H^{2}} \leq C|z|^{1 / 2}\|u\|_{L_{\partial}^{2}}^{1 / 2}\|H\|_{L_{\partial}^{2}}^{1 / 2}+C\left[\|f\|_{L^{2}}+\|H\|_{H_{\partial}^{1 / 2}}\right] .
$$


With the interpolation inequality 10.1 in [4] and the trace estimate (13) we get

$$
|z|^{1 / 2}\|u\|_{H^{1}} \leq C\left[\|f\|_{L^{2}}+\|H\|_{H_{\partial}^{1 / 2}}\right]+C|z|^{1 / 2}\|u\|_{H^{1}}^{1 / 2}\|H\|_{L_{\partial}^{2}}^{1 / 2},
$$

and so

$$
|z|^{1 / 2}\|u\|_{H^{1}} \leq C\left[\|f\|_{L^{2}}+\|H\|_{H_{\partial}^{1 / 2}}\right]+C|z|^{1 / 2}\|H\|_{L_{\partial}^{2}} .
$$

Now (15) implies

$$
|z|\|u\|_{L^{2}} \leq C|z|^{1 / 2}\left(\|u\|_{L_{\partial}^{2}}+\|H\|_{L_{\partial}^{2}}\right)+C\|f\|_{L^{2}},
$$

and so we get, estimating $\|u\|_{L_{\partial}^{2}}$ with (16) as before and using [2], that

$$
|z|\|u\|_{L^{2}}+\|u\|_{H^{2}} \leq C\left(\|f\|_{L^{2}}+|z|^{1 / 2}\|H\|_{H_{\partial}^{1 / 2}}\right) .
$$

Now we can apply this to our original problem by solving the equation $z G^{\prime}\left(p_{e}\right) \alpha+$ $\operatorname{div}\left(\rho_{e} u\right)=G^{\prime}\left(p_{e}\right) \widehat{f}_{2}$ for $\alpha$ to obtain $\alpha=z^{-1} \widehat{f}_{2}-\left(G^{\prime}\left(p_{e}\right) z\right)^{-1} \operatorname{div}\left(\rho_{e} u\right)$ and so we get

$$
\begin{array}{r}
z \rho_{e} u-\operatorname{div}(\mathbb{D}(u))+\nabla\left[z^{-1} \widehat{f_{2}}-\left(G^{\prime}\left(p_{e}\right) z\right)^{-1} \operatorname{div}\left(\rho_{e} u\right)\right] \\
\quad+g G^{\prime}\left(p_{e}\right)\left[z^{-1} \widehat{f}_{2}-\left(G^{\prime}\left(p_{e}\right) z\right)^{-1} \operatorname{div}\left(\rho_{e} u\right)\right] \mathbf{e}_{3}=\widehat{f}_{1}, \\
\mathbb{T}\left(u, z^{-1} \widehat{f}_{2}-\left(G^{\prime}\left(p_{e}\right) z\right)^{-1} \operatorname{div}\left(\rho_{e} u\right)\right) \cdot \mathbf{e}_{3}=H \text { at } x_{3}=0, u=0 \text { at } x_{3}=-h .
\end{array}
$$

With (17) this leads to

$$
\begin{aligned}
|z|\|u\|_{L^{2}}+\|u\|_{H^{2}} \leq C\left[\left\|\widehat{f}_{1}\right\|_{L^{2}}\right. & \left.+|z|^{-1}\left[\|u\|_{H^{2}}+\left\|\widehat{f}_{2}\right\|_{H^{1}}\right]\right] \\
& +C|z|^{1 / 2}\left(\|H\|_{H_{\partial}^{1 / 2}}+|z|^{-1}\left[\left\|\widehat{f}_{2}\right\|_{H_{\partial}^{1 / 2}}+\|u\|_{H_{\partial}^{3 / 2}}\right]\right),
\end{aligned}
$$

which for large $z$ implies

$$
|z|\|u\|_{L^{2}}+\|u\|_{H^{2}} \leq C\left[\left\|\widehat{f}_{1}\right\|_{L^{2}}+\left\|\widehat{f}_{2}\right\|_{H^{1}}\right]+C|z|^{1 / 2}\|H\|_{H_{\partial}^{1 / 2}} .
$$

Putting the results (18) for compressible and (14) for incompressible fluids together we get

$$
|z|\|u\|_{L^{2}}+\|u\|_{H^{2}} \leq C\left[\left\|\widehat{f}_{1}\right\|_{L^{2}}+\left\|G^{\prime}\left(p_{e}\right) \widehat{f}_{2}\right\|_{H^{1}}\right]+|z|^{1 / 2}\|H\|_{H_{\partial}^{1 / 2}},
$$

as $G^{\prime}\left(p_{e}\right)=0$ in the incompressible case, and in the compressible case $\left(G^{\prime}\left(p_{e}\right)\right)^{-1}$ is bounded on $[-h, 0]$, and so our lemma is proved.

If we add the variable $\varphi$ on $\left[0, l_{1}\right] \times\left[0, l_{2}\right]$ and the equations

$$
H=\left(p_{e}\right)_{x_{3}}(0) \varphi \mathbf{e}_{3}, \quad z \varphi=u_{3}+h,
$$

to $(11)$ we get

LEMma 6. For $\widehat{f}_{1} \in L^{2}, \widehat{f}_{2}, \alpha \in H^{1}, h \in H_{\partial}^{\mu}, u \in H^{2}$ fulfilling the equation (11) together with (19) we have, if $z$ is sufficiently large

$$
\begin{aligned}
|z|\|u\|_{L^{2}}+\|u\|_{H^{2}}+|z|\left\|G^{\prime}\left(p_{e}\right) \alpha\right\|_{H^{1}}+|z|\|\varphi\|_{H_{\partial}^{\mu}} & \\
& \leq C\left[\left\|\widehat{f}_{1}\right\|_{L^{2}}+\left\|G^{\prime}\left(p_{e}\right) \widehat{f}_{2}\right\|_{H^{1}}+\|h\|_{H_{\partial}^{\mu}}\right]
\end{aligned}
$$

for $\mu \in[1 / 2,3 / 2]$. 
Proof. From Lemma 5 we get

$$
|z|\|u\|_{L^{2}}+\|u\|_{H^{2}} \leq C\left[\left\|\widehat{f}_{1}\right\|_{L^{2}}+\left\|G^{\prime}\left(p_{e}\right) \widehat{f}_{2}\right\|_{H^{1}}\right]+|z|^{1 / 2}\left\|z^{-1}\left(h+u_{3}\right)\right\|_{H_{\partial}^{1 / 2}},
$$

and so

$$
|z|\|u\|_{L^{2}}+\|u\|_{H^{2}} \leq C\left[\left\|\widehat{f}_{1}\right\|_{L^{2}}+\left\|G^{\prime}\left(p_{e}\right) \widehat{f}_{2}\right\|_{H^{1}}+\|h\|_{H_{\partial}^{1 / 2}}\right],
$$

from which we easily get the estimates for the other variables using the equations.

This estimate provides the most important building block for using the theory of analytic semigroups for our equation. For the compressible case this is indeed fairly easy in view of [7]. We can then use

$$
\left\{(u, \alpha, \varphi): u \in L_{2}, \alpha \in H^{1}, \varphi \in H_{\partial}^{\mu}\right\}
$$

$(1 / 2 \leq \mu \leq 3 / 2)$ as the basic space, with the subspace of all elements in

$$
\left\{(u, \alpha, \varphi): u \in H^{2}, \alpha \in H^{1}, \varphi \in H_{\partial}^{\mu}\right\}
$$

fulfilling the boundary conditions (6) as the domain of definition, and the definition of the operator is clear from the form of the estimates.

For incompressible flows first let

$$
B=\left\{(u, \varphi): u \in B_{1}, \varphi \in H_{\partial}^{\mu}\right\} .
$$

(For $B_{1}$ see (7).) As the domain of definition $D(A)$ of our operator we take

$$
\left\{(u, \varphi) \in B: u \in H^{2}, u=0 \text { at } x_{3}=-h, \mathbb{D}(u) \cdot \mathbf{n}=\omega \mathbf{n} \text { for an } \omega \in H_{\partial}^{1 / 2}\right\} .
$$

Now we need to see that $D(A)$ is dense in $B$. As $u$ and $\varphi$ are not really related in $B$ and $D(A)$, we only need to show that

$$
D_{1}=\left\{u \in B_{1} \cap H^{2}: u=0 \text { at } x_{3}=-h, \mathbb{D}(u) \cdot \mathbf{n}=\omega \mathbf{n} \text { for an } \omega \in H_{\partial}^{1 / 2}\right\}
$$

is dense in $B_{1}$. Assume $u \in B_{1}$ and

$$
\int_{\mathcal{R}^{0}} u \cdot v d x=0
$$

for all $v \in D_{1}$. By Lemma 4 there is a function $w \in D_{1}$ and a $g \in H^{1}$ such that $u=-\operatorname{div}(\mathbb{D}(w))+\nabla g, \operatorname{div}(w)=0, w=0$ at $x_{3}=-h$ and $-\mathbb{D}(w) \cdot \mathbf{e}_{3}+g \mathbf{e}_{3}=0$ at $x_{3}=0$. Then

$$
\begin{array}{rl}
0=\int_{\mathcal{R}^{0}}(-\operatorname{div}(\mathbb{D}(w))+\nabla g) \cdot v & d x=\int_{\mathcal{R}^{0}}\left(\mathbb{D}_{i j}(w)-\delta_{i j} g\right) v_{i x_{j}} d x \\
=\int_{\mathcal{R}^{0}} \mathbb{D}_{i j}(w) v_{i x_{j}}-\operatorname{div}(v) g d x=\int_{\mathcal{R}^{0}} \mathbb{D}_{i j}(w) v_{i x_{j}} d x .
\end{array}
$$

As $w \in D_{1}$ we can choose $v=w$ and obtain $\mathbb{D}(w)=0$, and due to the boundary condition at $x_{3}=-h$ we have $w=0$. So $g=0$ at $x_{3}=0$, and $u=\nabla g$, so by the definition of $B_{1}$ we have $u=0$. This proves that $D(A)$ is dense in $B$.

The reader will have noticed that as is usual for incompressible flows the pressure has disappeared from the space. This makes the definition of the operator a little bit tricky. For $(u, \varphi) \in D(A)$ let

$$
A(u, \varphi)=\left(-\operatorname{div} \mathbb{D}(u)+\nabla \alpha, u_{3}\right)
$$


where $\alpha$ is given by the conditions

$$
\alpha=p_{\text {ex }}(0) \varphi+\mathbf{e}_{3} \cdot \mathbb{D}(u) \cdot \mathbf{e}_{3}
$$

on $\left\{x_{3}=0\right\}$ and

$$
\int_{\mathcal{R}^{0}}(-\operatorname{div} \mathbb{D}(u)+\nabla \alpha) \nabla \Phi d x=0
$$

for all $\Phi \in H^{1}$ with $\Phi=0$ at $x_{3}=0$. With these definitions it is easy to verify the conditions of Theorem 2.1 in [4], which gives us Theorem 1. The consideration for incompressible flows is in part parallel to that done in Beale's paper [3].

4. The evolution of the linearized system. In this section we first formulate a conservation law. From here all our solutions are real-valued.

LEMMA 7. Let $I$ be a real interval and assume $u, \alpha, \varphi$ with $u \in C^{0}\left(I, H^{2}\right) \cap C^{1}\left(I, L^{2}\right)$, $\alpha \in C^{1}\left(I, H^{1}\right), \varphi \in C^{1}\left(I, L_{\partial}^{2}\right)$ solve the system of equations (5) with the boundary conditions (6). Then with

$$
E(u, \alpha, \varphi)(t)=\frac{1}{2}\left[-p_{e x_{3}}(0) \int_{\mathcal{R}^{0} \cap\left\{x_{3}=0\right\}} \varphi^{2} d \sigma+\int_{\mathcal{R}^{0}} \rho_{e} u^{2}+\rho_{e}^{-1} G^{\prime}\left(p_{e}\right) \alpha^{2} d x\right]
$$

we have

$$
\frac{d E}{d t}=-\int_{\mathcal{R}^{0}} \mathbb{D}_{i j}(u) u_{i x_{j}} d x .
$$

Proof. Apply Lemma 3 with $f_{1}=-\rho_{e} u_{t}$, and $f_{2}=-G^{\prime}\left(p_{e}\right) \alpha_{t}, H=p_{e x_{3}}(0) \varphi \mathbf{e}_{3}$, also assuming the solutions are real. This gives us

$$
\int_{\mathcal{R}^{0}} \mathbb{D}_{i j}(u) u_{i x_{j}} d x=\int_{\mathcal{R}^{0} \cap\left\{x_{3}=0\right\}} p_{e x_{3}}(0) \varphi u_{3} d \sigma-\int_{\mathcal{R}^{0}} \rho_{e} u_{t} u+G^{\prime}\left(p_{e}\right) \rho_{e}^{-1} \alpha_{t} \alpha d x,
$$

and as $\varphi_{t}=u_{3}$ we immediately get our claim.

We need to define a family of operators before we can go on.

Definition 8. Let $\mu \geq 0$. For $f \in H_{\partial}^{\mu}$ with

$$
f=\sum_{k, m=-\infty}^{\infty} a_{k m} \exp \left(2 \pi i\left(k \frac{x_{1}}{l_{1}}+m \frac{x_{2}}{l_{2}}\right)\right)
$$

let

$$
D_{\mu} f=\sum_{k, m=-\infty}^{\infty}\left(1+k^{2}+m^{2}\right)^{\mu / 2} a_{k m} \exp \left(2 \pi i\left(k \frac{x_{1}}{l_{1}}+m \frac{x_{2}}{l_{2}}\right)\right) .
$$

Then it is clear that $D_{\mu}: H_{\partial}^{\alpha+\mu} \rightarrow H_{\partial}^{\alpha}$ is an isomorphism for $\alpha \geq 0$. Also we can apply $D_{\mu}$ to functions defined on $\left(0, l_{1}\right) \times\left(0, l_{2}\right) \times(-h, 0)$ by fixing $x_{3}$ and applying $D_{\mu}$ with respect to $x_{1}$ and $x_{2}$. We also denote this operator by $D_{\mu}$.

Lemma 7 now implies that $E(u, \alpha, \varphi)$ is a decreasing function of time. We can differentiate the equations (5) and (6) with respect to time and obtain that $E\left(u_{t}, \alpha_{t}, \varphi_{t}\right)$ is also decreasing, and due to the fact that our operator generates an analytic semigroup time derivatives of arbitrary order exist for $t>0$. Likewise all coefficients occurring in the equation are independent of $x_{1}$ and $x_{2}$, so if $(u, \alpha, \varphi)$ is a sufficiently regular solution 
of our equation, then $\left(D_{\mu} u, D_{\mu} \alpha, D_{\mu} \varphi\right)$ also is a solution, and so $E\left(D_{\mu} u, D_{\mu} \alpha, D_{\mu} \varphi\right)$ as well as $E\left(D_{\mu} u_{t}, D_{\mu} \alpha_{t}, D_{\mu} \varphi_{t}\right)$ are decreasing.

This means that if $E\left(D_{\mu} u, D_{\mu} \alpha, D_{\mu} \varphi\right)$ is small at the beginning, it will always be small, and if $E\left(D_{\mu} u_{t}, D_{\mu} \alpha_{t}, D_{\mu} \varphi_{t}\right)$ is small at the beginning, it will always be small also. So to obtain slow decay we want to construct a big initial value with a small time derivative. To do this consider the equation

$$
\begin{aligned}
-\operatorname{div}\left(\mathbb{T}\left(u_{0}, \alpha_{0}\right)\right) & =-\alpha_{0} G^{\prime}\left(p_{e}\right) g \mathbf{e}_{3}, \\
\operatorname{div}\left(\rho_{e} u_{0}\right) & =0,
\end{aligned}
$$

with the boundary condition $\mathbb{T}\left(u_{0}, \alpha_{0}\right) \cdot \mathbf{n}=H$ at the top and $u_{0}=0$ at the bottom. We already proved the solvability of this equation. So we first give ourselves $\varphi_{0}$ and then solve our equation with $H=p_{e x_{3}}(0) \mathbf{e}_{3} \varphi_{0}$ to obtain $u_{0}$ and $\alpha_{0}$. This means that if we take these as initial values for a solution we have $u_{t}(0)=0, \alpha_{t}(0)=0$, and $\varphi_{t}(0)=u_{3}(0)$. From here we will use $\left\|D_{\mu} h\right\|_{L_{\partial}^{2}}$ as the norm $\|h\|_{H_{\partial}^{\mu}}$ of $h$. We have with $\mu \geq 0$ by Lemma 4

$$
\left\|u_{3}(0) \mid\left\{x_{3}=0\right\}\right\|_{H_{\partial}^{1+\mu}} \leq C\left\|u_{3}(0)\right\|_{H^{3 / 2+\mu}} \leq C\left\|\varphi_{0}\right\|_{H_{\partial}^{\mu}},
$$

and so the mapping $\varphi_{0} \rightarrow u_{3}(0) \mid\left\{x_{3}=0\right\}$ is compact as a mapping from $H_{\partial}^{\mu}$ into itself. Therefore we can, given $\varepsilon>0$, choose $\varphi_{0}$ in such a way that $\left\|\varphi_{0}\right\|_{H_{\partial}^{\mu}}=1$ and $\left\|u_{3}(0) \mid\left\{x_{3}=0\right\}\right\|_{H_{\partial}^{\mu}} \leq \varepsilon$. As $\varphi_{t}(0)=u_{3}(0) \mid\left\{x_{3}=0\right\}$, we have $\left\|\varphi_{t}(0)\right\|_{H_{\partial}^{\mu}} \leq \varepsilon$. Now also

$$
\begin{aligned}
& \int_{\mathcal{R}^{0}} \rho_{e}\left(D_{\mu} u_{t}\right)^{2}(t) d x+\int_{\mathcal{R}^{0}} G^{\prime}\left(p_{e}\right) \rho_{e}^{-1}\left(D_{\mu} \alpha_{t}\right)^{2}(t) d x \\
& \quad-p_{\text {ex }}(0) \int_{\left\{x_{3}=0\right\} \cap \mathcal{R}^{0}}\left(D_{\mu} \varphi_{t}\right)^{2}(t) d \sigma \leq 0+0-p_{e x_{3}}(0) \int_{\left\{x_{3}=0\right\} \cap \mathcal{R}^{0}}\left(D_{\mu} \varphi_{t}\right)^{2}(0) d \sigma,
\end{aligned}
$$

and so

$$
-p_{e x_{3}}(0) \int_{\left\{x_{3}=0\right\} \cap \mathcal{R}^{0}}\left(D_{\mu} \varphi_{t}\right)^{2}(t) d \sigma \leq-p_{e x_{3}}(0) \int_{\left\{x_{3}=0\right\} \cap \mathcal{R}^{0}}\left(D_{\mu} \varphi_{t}\right)^{2}(0) d \sigma,
$$

which gives us $\left\|\varphi_{t}(t)\right\|_{H_{\partial}^{\mu}} \leq\left\|\varphi_{t}(0)\right\|_{H_{\partial}^{\mu}} \leq \varepsilon$ for all times $t \geq 0$. This implies

$$
\begin{aligned}
\|\varphi(t)\|_{H_{\partial}^{\mu}}=\left\|\varphi(0)+\int_{0}^{t} \varphi_{t}(\tau) d \tau\right\|_{H_{\partial}^{\mu}} \geq\|\varphi(0)\|_{H_{\partial}^{\mu}} & -\int_{0}^{t}\left\|\varphi_{t}(\tau)\right\|_{H_{\partial}^{\mu}} d \tau \\
& \geq 1-\varepsilon t=(1-\varepsilon t)\|\varphi(0)\|_{H_{\partial}^{\mu}} .
\end{aligned}
$$

Now for $\beta_{1} \in[\mu, \mu+3 / 2], \beta_{2} \in[\mu, \mu+1 / 2]$ let

$$
\left\|\left(u_{0}, \alpha_{0}, \varphi_{0}\right)\right\|_{\mathcal{N}}=\left\|u_{0}\right\|_{H^{\beta_{1}}}+\left\|\alpha_{0}\right\|_{H^{\beta_{2}}}+\left\|\varphi_{0}\right\|_{H_{\partial}^{\mu}}
$$

Then we have, as we just saw,

$$
\|(u, \alpha, \varphi)(0)\|_{\mathcal{N}} \leq C\left\|\varphi_{0}\right\|_{H_{\partial}^{\mu}},
$$

and, if $\varepsilon t<1$,

$$
\begin{aligned}
\left\|\left(u, G^{\prime}\left(p_{e}\right) \alpha, \varphi\right)(t)\right\|_{\mathcal{N}} \geq\|\varphi(t)\|_{H_{\partial}^{\mu}} \geq(1-\varepsilon t)\|\varphi(0)\|_{H_{\partial}^{\mu}} \\
\quad \geq C^{-1}(1-\varepsilon t)\|(u, \alpha, \varphi)(0)\|_{\mathcal{N}} .
\end{aligned}
$$


Together with our assumption of uniform exponential decay this implies

$$
C^{\prime} e^{-\gamma t}\left\|\left(u, G^{\prime}\left(p_{e}\right) \alpha, \varphi\right)(0)\right\|_{\mathcal{N}} \geq\|(u, \alpha, \varphi)(t)\|_{\mathcal{N}} \geq C^{-1}(1-\varepsilon t)\|(u, \alpha, \varphi)(0)\|_{\mathcal{N}},
$$

and so $1-\varepsilon t \leq C C^{\prime} e^{-\gamma t}$ for all $t>0, \varepsilon>0$, which is a contradiction. This proves the part of the claim about lack of decay.

Now we have to prove there is any decay at all. This is the content of the following lemma.

Lemma 9. Let $u, \alpha, \varphi$ with $u \in C^{0}\left([0, \infty), H^{2}\right) \cap C^{1}\left([0, \infty), L^{2}\right), \alpha \in C^{1}\left([0, \infty), H^{1}\right)$, $\varphi \in C^{1}\left([0, \infty), L_{\partial}^{2}\right)$ solve the system of equations (5) with the boundary conditions (6), and let $\mathcal{C}(t)=0$. Then $u(t), G^{\prime}\left(p_{e}\right) \alpha(t)$ converge to zero weakly in $L^{2}$, and $\varphi(t)$ does so weakly in $L_{\partial}^{2}$.

Proof. From Lemma 7 we obtain by integration that

$$
E(u, \alpha, \varphi)\left(T_{1}\right)-E(u, \alpha, \varphi)\left(T_{2}\right)=\int_{T_{1}}^{T_{2}} \int_{\mathcal{R}^{0}}|\mathbb{D} u|^{2} d x d t
$$

for all $T>0$. The same is true for the time derivatives. As a result

$$
\int_{T}^{T+1} \int_{\mathcal{R}^{0}}|\mathbb{D} u|^{2}+\left|\mathbb{D} u_{t}\right|^{2} d x d t \rightarrow 0
$$

and so

$$
\int_{\mathcal{R}^{0}}|\mathbb{D} u|^{2} d x \rightarrow 0
$$

as $t \rightarrow \infty$. As $\|u\|_{L_{2}}$ is bounded, we can select a sequence of times $t_{k}$ for which $u\left(t_{k}\right) \rightarrow u_{\infty}$, and now $\mathbb{D} u_{\infty}=0$, so by this and the boundary condition at the bottom we get $u_{\infty}=0$. So $u(t)$ actually converges weakly to zero in $L_{2}$. In the same way we can show $u_{t}$ converges weakly to zero in $L_{2}$. Multiplying the first equation by an arbitrary function vector $v \in C^{\infty}(\mathcal{R})$ which is periodic in the $x_{1}$ and $x_{2}$ directions and zero at $x_{3}=-h$, and integrating it over $\mathcal{R}^{0}$ we get $I_{1}-I_{2}+I_{3}=0$ with

$$
I_{1}=\int_{\mathcal{R}^{0}} \rho_{e} u_{t}(t) v d x, I_{2}=\int_{\mathcal{R}^{0}} \operatorname{div}(\mathbb{T}(u(t), \alpha(t))) v d x, I_{3}=\int_{\mathcal{R}^{0}} g G^{\prime}\left(p_{e}\right) \alpha(t) v_{3} d x .
$$

Now $\lim _{t \rightarrow \infty} I_{1}=0$, and if $\left(0, \alpha_{\infty}, \varphi_{\infty}\right)$ denotes any vector function which is a weak limit of $\left(u\left(t_{k}\right), \alpha\left(t_{k}\right), \varphi\left(t_{k}\right)\right)$ for a sequence $t_{k} \rightarrow \infty$, then

$$
\lim _{t \rightarrow \infty} I_{3}=\int_{\mathcal{R}^{0}} g G^{\prime}\left(p_{e}\right) \alpha_{\infty} v_{3} d x
$$

and, integrating by parts,

$$
I_{2}=\int_{\mathcal{R}^{0}}-\mathbb{T}_{i j}(u(t), \alpha(t)) v_{i x_{j}} d x+\int_{\mathcal{R}^{0} \cap\left\{x_{3}=0\right\}} \mathbb{T}_{i j}(u(t), \alpha(t)) v_{i} n_{j} d \sigma=I_{21}+I_{22} .
$$

Now

$$
I_{21}=\int_{\mathcal{R}^{0}}-\mathbb{D}_{i j}(u(t)) v_{i x_{j}}+\alpha(t) \operatorname{div}(v) d x \rightarrow \int_{\mathcal{R}^{0}} \alpha_{\infty} \operatorname{div}(v) d x,
$$

as $\mathbb{D} u \rightarrow 0$, and by the boundary condition

$$
I_{22}=\int_{\mathcal{R}^{0} \cap\left\{x_{3}=0\right\}} p_{e x_{3}}(0) \varphi(t) v_{3} d \sigma \rightarrow \int_{\mathcal{R}^{0} \cap\left\{x_{3}=0\right\}} p_{e x_{3}}(0) \varphi_{\infty} v_{3} d \sigma .
$$


So we get

$$
\int_{\mathcal{R}^{0}}-\alpha_{\infty} \operatorname{div}(v)+g G^{\prime}\left(p_{e}\right) \alpha_{\infty} v_{3} d x-\int_{\mathcal{R}^{0} \cap\left\{x_{3}=0\right\}} p_{e x_{3}}(0) \varphi_{\infty} v_{3} d \sigma=0 .
$$

This implies that $\alpha_{\infty}$ only depends on $x_{3}$ and fulfils the differential equation $\left(\alpha_{\infty}\right)_{x_{3}}=$ $-g G^{\prime}\left(p_{e}\right) \alpha_{\infty}$, and so it has a limit at $x_{3}=0$, which we denote by $\alpha_{\infty}(0)$. Due to this equation $\alpha_{\infty}$ also cannot change sign in $[-h, 0]$. Integrating by parts we get

$$
\int_{\mathcal{R}^{0} \cap\left\{x_{3}=0\right\}}\left(\alpha_{\infty}(0)+p_{\text {ex }}(0) \varphi_{\infty}\right) v_{3} d \sigma=0 .
$$

So $\alpha_{\infty}(0)=-p_{e x_{3}}(0) \varphi_{\infty}$, and $\varphi_{\infty}$ is a constant. As $p_{e x_{3}}(0)<0$ both $\varphi_{\infty}$ and $\alpha_{\infty}(0)$ have the same sign, and the sign of $\alpha_{\infty}$ cannot change in $[-h, 0]$, so both $\varphi_{\infty}$ and $\alpha_{\infty}$ must have the same sign where they are defined, due to the differential equation (4). Also as $\mathcal{C}(t)=0$ we have

$$
\int_{\mathcal{R}^{0}} G^{\prime}\left(p_{e}\right) \alpha_{\infty} d x+\rho_{e}(0) \int_{\mathcal{R}^{0} \cap\left\{x_{3}=0\right\}} \varphi_{\infty} d \sigma=0 .
$$

This is only possible if $G^{\prime}\left(p_{e}\right) \alpha_{\infty}=0$ and $\varphi_{\infty}=0$, as they both have the same sign. So we have shown that, weakly in $L^{2}$, our solutions converge to zero.

5. The linearization of the equation. As we will see in the last section, the following approach to the linearization of equations is sufficient to draw conclusions about the lack of exponential decay of the non-linear equations.

We consider a one-parameter family $(v(\varepsilon), p(\varepsilon), f(\varepsilon))$ of solutions of our equations, which depend smoothly on their variables, and $(v(0), p(0), f(0))=\left(0, p_{e}, 0\right)$.

We do not want to prove the existence of such families, but by a careful analysis of "short term" existence proofs one can see that such solutions, as the initial value approaches an equilibrium state, exist for times converging to infinity. Then it is technically difficult to show that the solutions depend differentiably on a parameter, if the initial values do, but does not require any tools beyond what is available in [5] and [3].

Now the linearization is the equation obtained by differentiating (1) and (2) with respect to $\varepsilon$ at $\varepsilon=0$, where we denote the derivatives of these functions by

$$
u=\frac{\partial v}{\partial \varepsilon}(0), \quad \alpha=\frac{\partial p}{\partial \varepsilon}(0), \quad \varphi=\frac{\partial f}{\partial \varepsilon}(0),
$$

which are defined on $\mathcal{R}^{0}, \mathcal{R}^{0}$, and $\left[0, l_{1}\right] \times\left[0, l_{2}\right]$ respectively. We can take these derivatives of the differential equations themselves in the interior directly, but have to think a little more about the boundary conditions. As an example let us consider the expression $\rho v_{t}$. Away from the top boundary we have

$$
\frac{\partial}{\partial \varepsilon}\left(\rho v_{t}\right)=\rho \frac{\partial}{\partial \varepsilon}\left(v_{t}\right)+v_{t} \frac{\partial}{\partial \varepsilon}(\rho)=\rho_{e} \frac{\partial^{2}}{\partial \varepsilon \partial t}(v)+0=\rho_{e} u_{t}
$$

using $v(t, 0)=0$. In a similar way we obtain

$$
\rho_{e} u_{t}-\operatorname{div}(\mathbb{D}(u))+\nabla \alpha=-g G^{\prime}\left(p_{e}\right) \alpha \mathbf{e}_{3},
$$

and

$$
G^{\prime}\left(p_{e}\right) \alpha_{t}+\operatorname{div}\left(\rho_{e} u\right)=0
$$


As $f \rightarrow 0$ as $\varepsilon \rightarrow 0$ this is actually valid in all of $\mathcal{R}^{0}$.

We cannot do this as easily for the boundary conditions, but have to transform our variable domains to a fixed one, which we can of course choose as $\mathcal{R}^{0}$. Let us consider the effects of such transformations on the linearization. So let $T_{t}(y, \varepsilon)$ for fixed $t, \varepsilon$ be a diffeomorphism from $\mathcal{R}^{0}$ to $\Omega_{t}^{\varepsilon}$, and let $T_{t}(y, 0)=y$, and assume $T$ is sufficiently smooth as a function of all its variables. This is true for the transformation describing the transition from Euler to Lagrange coordinates, and also others. Then if $h(x, t, \varepsilon)$ for fixed $\varepsilon, t$ is defined on $\Omega_{t}^{\varepsilon}$, and $\widetilde{h}(y, t, \varepsilon)=h\left(T_{t}(y, \varepsilon), t, \varepsilon\right)$, for a function $h$, and $h(x, t, 0)=h_{e}(x)$, then

$$
\begin{aligned}
\frac{\partial \widetilde{h}}{\partial \varepsilon}(y, t, 0)=h_{x_{k}}(y, t, 0) \frac{\partial\left(T_{t}\right)_{k}}{\partial \varepsilon}(y, 0)+\frac{\partial h}{\partial \varepsilon}\left(T_{t}(y, 0), t, 0\right) & \\
& =h_{e x_{k}}(y) \frac{\partial\left(T_{t}\right)_{k}}{\partial \varepsilon}+\frac{\partial h}{\partial \varepsilon}(y, t, 0)
\end{aligned}
$$

SO

$$
\frac{\partial \widetilde{h}}{\partial \varepsilon}(y, t, 0)=h_{e x_{k}}(y) \frac{\partial\left(T_{t}\right)_{k}}{\partial \varepsilon}+\frac{\partial h}{\partial \varepsilon}(y, t, 0) .
$$

So for any variable whose value at equilibrium is independent of the space variables, the linearization is independent of the coordinate system which we are using.

For our considerations we want to use the coordinate transformation

$$
T_{t}\left(y_{1}, y_{2}, y_{3}, \varepsilon\right)=\left(y_{1}, y_{2}, y_{3}+\frac{y_{3}+h}{h} f\left(y_{1}, y_{2}, t, \varepsilon\right)\right),
$$

which has the properties we just required, and

$$
\frac{\partial T_{t}}{\partial \varepsilon}(y, t, 0)=\left(0,0, \frac{y_{3}+h}{h} \varphi\left(y_{1}, y_{2}, t, 0\right)\right)
$$

which equals $\varphi \mathbf{e}_{3}$ on the upper boundary. Let $x=T_{t}(y)$. With

$$
\frac{\partial x_{k}}{\partial y_{m}}=\frac{\partial T_{k}}{\partial y_{m}} \quad \text { and } \quad \frac{\partial y_{k}}{\partial x_{m}}=\frac{\partial T_{k}^{-1}}{\partial x_{m}}
$$

then in these coordinates the boundary conditions transform to

$$
\widetilde{\mathbb{T}}(y, t) \cdot \mathbf{n}(y, t)=\mathrm{p}_{0} \mathbf{n}(y, t),
$$

where $\mathbb{D}$ is transformed into

$$
\widetilde{\mathbb{D}}(\widetilde{v})=\left[\widetilde{\mathbb{D}}_{i j}(\widetilde{v})\right]=\left[\nu_{1}\left(\frac{\partial y_{k}}{\partial x_{j}} \frac{\partial \widetilde{v}_{i}}{\partial y_{k}}+\frac{\partial y_{k}}{\partial x_{i}} \frac{\partial \widetilde{v}_{j}}{\partial y_{k}}\right)+\left(\nu_{2}-\nu_{1}\right) \delta_{i j} \frac{\partial y_{k}}{\partial x_{l}} \frac{\partial \widetilde{v}_{l}}{\partial y_{k}}\right]
$$

where $\widetilde{v}(y, t, \varepsilon)=v\left(T_{t}(y, \varepsilon), t, \varepsilon\right)$, and of course $\widetilde{\mathbb{T}}(y, t)=\widetilde{\mathbb{D}}(\widetilde{v})-\delta_{i j} \widetilde{p}$ with $\widetilde{p}(y, t, \varepsilon)=$ $p\left(T_{t}(y, \varepsilon), t, \varepsilon\right)$. For $\varepsilon=0$ the transformation $T_{t}$ is the identity, and so, as

$$
\frac{\partial}{\partial \varepsilon}(\widetilde{\mathbb{T}}(\widetilde{v}, \widetilde{p}) \cdot \mathbf{n})=-\frac{\partial}{\partial \varepsilon}\left(\mathrm{p}_{0} \mathbf{n}\right),
$$

we also get, as $\mathrm{p}_{0}$ is constant, that

$$
\frac{\partial}{\partial \varepsilon}(\widetilde{\mathbb{T}}(\widetilde{v}, \widetilde{p})) \cdot \mathbf{n}+\widetilde{\mathbb{T}}(\widetilde{v}, \widetilde{p}) \cdot \frac{\partial \mathbf{n}}{\partial \varepsilon}=-\mathrm{p}_{0} \frac{\partial \mathbf{n}}{\partial \varepsilon} .
$$


Now $\widetilde{\mathbb{T}}_{i j}(\widetilde{v}, \widetilde{p})(y, t, 0)=-\delta_{i j} p_{e}(0)=-\delta_{i j} \mathrm{p}_{0}$ due to the boundary condition, and so

$$
0=\frac{\partial}{\partial \varepsilon}(\widetilde{\mathbb{T}}(\widetilde{v}, \widetilde{p})) \cdot \mathbf{n}=\frac{\partial}{\partial \varepsilon}(\widetilde{\mathbb{D}}(\widetilde{v})) \cdot \mathbf{n}-\frac{\partial}{\partial \varepsilon}(\widetilde{p}) \mathbf{n} .
$$

Using (21) this is easily seen to imply

$$
\mathbb{T}(u, \alpha) \cdot \mathbf{e}_{3}=\mathbb{D}(u) \cdot \mathbf{e}_{3}-\alpha \mathbf{e}_{3}=\left(p_{e}\right)_{x_{3}} \varphi \mathbf{e}_{3} .
$$

Now we have derived the boundary conditions (6) and the first two equations of (5). Now the last of these equations follows by the differentiation of (3) with respect to $\varepsilon$ after transforming to a fixed domain and keeping the relationship between the variables with and without $\sim$ in mind.

This finishes the computation of the linearization.

6. Decay and the linearization. As the equilibrium solution and the solution approaching it have different domains of definition, it needs to be clarified what concept of distance between these two functions one might want to use. This is, however, not as problematic as it may seem. As we can extend this equilibrium solution a bit above zero, it is still defined on the set $\Omega_{t}^{0}$, provided $f$ is small, and then we can use function spaces for this domain for defining the distance.

The following discussion is for the compressible case alone. At the end we will indicate the changes necessary in the incompressible case.

Let us first define what is meant by exponential decay in this case. To this end we introduce the spaces

$$
B_{1}\left(\Omega_{t}^{0}\right)=\left\{(v, p): v \in H^{\beta_{1}}\left(\Omega_{t}^{0}\right), p \in H^{\beta_{2}}\left(\Omega_{t}^{0}\right)\right\}
$$

for velocity and pressure, and

$$
\widehat{B}_{1}=\left\{f: f \in H^{\mu}\left(\left[0, l_{1}\right] \times\left[0, l_{2}\right]\right)\right\}
$$

for the boundary, where $\mu \geq 0$ is real but $\beta_{1} \in[\mu, \mu+3 / 2], \beta_{2} \in[\mu, \mu+1 / 2]$ now must be integers.

As we cannot necessarily expect to even have a solution for initial values in these spaces, as the functions are not necessarily regular enough, we assume there are two more Sobolev spaces, $B_{2}\left(\Omega_{t}^{0}\right)$ and $\widehat{B}_{2}$ dense in $B_{1}\left(\Omega_{t}^{0}\right)$ and $\widehat{B}_{1}$, and chosen so that if $\left(u_{0}, p_{0}\right) \in B_{2}\left(\Omega_{0}^{0}\right)$, and $f_{0} \in \widehat{B}_{2}$, a solution will exist for some time, and the length of the interval of existence goes to infinity as the distance between the equilibrium and the initial values goes to zero in $B_{2}\left(\Omega_{0}^{0}\right), \widehat{B}_{2}$. Now by uniform exponential convergence to the equilibrium state we mean that in addition to the assumptions made so far there are two constants $0<\gamma, C<\infty$ such that

$$
\begin{aligned}
\left\|(v, p)(t)-\left(0, p_{e}(y)\right)\right\|_{B_{1}\left(\Omega_{t}^{0}\right)} & +\|f(t)\|_{\widehat{B}_{1}} \\
\leq & C e^{-\gamma t}\left(\left\|(v, p)(0)-\left(0, p_{e}(y)\right)\right\|_{B_{1}\left(\Omega_{0}^{0}\right)}+\|f(0)\|_{\widehat{B}_{1}}\right),
\end{aligned}
$$

for all solutions $(v, p, f)$ sufficiently close to this equilibrium in the Banach spaces $B_{2}$ and $\widehat{B}_{2}$, and which also have the same mass. Now we want to see what consequences we can draw from the estimate (22) for the linearization of our equation. 
So now assume $\left(u_{0}, \alpha_{0}\right) \in B_{2}\left(\mathcal{R}^{0}\right)$ and $\varphi_{0} \in \widehat{B}_{2}$ and

$$
\int_{\mathcal{R}^{0}} G^{\prime}\left(p_{e}\right) \alpha_{0} d x+\rho_{e}(0) \int_{\mathcal{R}^{0} \cap\left\{x_{3}=0\right\}} \varphi_{0} d x=0 .
$$

This is the type of initial values we have considered in Sec. 3 and 4 . Then we might think we can use $\left(\varepsilon u_{0}, \varepsilon \alpha_{0}+p_{e}, \varepsilon \varphi_{0}\right)$ as a family of initial values for the non-linear equation giving rise to a family of solutions. They do, however, not all have the same mass. To remedy that we can add a function $d(\varepsilon)$ to $\varphi_{0}$ to get $\left(\varepsilon u_{0}, \varepsilon \alpha_{0}+p_{e}, \varepsilon \varphi_{0}+d(\varepsilon)\right)$. As it will turn out we have $d^{\prime}(0)=0$, and so

$$
\frac{\partial}{\partial \varepsilon}\left(\varepsilon u_{0}, \varepsilon \alpha_{0}+p_{e}, \varepsilon \varphi_{0}+d(\varepsilon)\right)=\left(u_{0}, \alpha_{0}, \varphi_{0}\right)
$$

at $\varepsilon=0$. Now let us determine $d$.

We have

$$
M=\int_{0}^{l_{1}} \int_{0}^{l_{2}} \int_{-h}^{\varepsilon \varphi_{0}\left(x_{1}, x_{2}\right)+d(\varepsilon)} G\left(p_{e}\left(x_{3}\right)+\varepsilon \alpha_{0}(x)\right) d x_{3} d x_{2} d x_{1} .
$$

Now we have, if we consider $M$ as a function of $d$,

$$
\frac{\partial M}{\partial d}=\int_{0}^{l_{1}} \int_{0}^{l_{2}} G\left(p_{e}\left(\varepsilon \varphi_{0}\left(x_{1}, x_{2}\right)+d\right)+\varepsilon \alpha_{0}\left(x_{1}, x_{2}, \varepsilon \varphi_{0}\left(x_{1}, x_{2}\right)+d\right)\right) d x_{2} d x_{1},
$$

and if we have chosen $B_{2}$ and $\widehat{B}_{2}$ in such a way that they are continuously embedded in $L^{\infty}$, then $\frac{\partial M}{\partial d}>0$ for small $\varepsilon$. This means that such $d$ exist and depend differentiably on $\varepsilon$. Then for the solution $M$ is independent of $\varepsilon$ and $t$, and so, differentiating (24) with respect to $\varepsilon$ at $\varepsilon=0$, we deduce that

$$
\int_{0}^{l_{1}} \int_{0}^{l_{2}} G\left(\mathrm{p}_{0}\right)\left[\varphi_{0}\left(x_{1}, x_{2}\right)+d^{\prime}(0)\right] d x_{2} d x_{1}+\int_{0}^{l_{1}} \int_{0}^{l_{2}} \int_{-h}^{0} G^{\prime}\left(p_{e}(x)\right) \alpha_{0} d x_{3} d x_{2} d x_{1}
$$

equals zero. Therefore due to (23) this implies

$$
0=d^{\prime}(0) \int_{0}^{l_{1}} \int_{0}^{l_{2}} G\left(\mathrm{p}_{0}\right) d x_{2} d x_{1}
$$

and so $d^{\prime}(0)=0$.

If we now use the solutions with the initial values $\left(\varepsilon u_{0}, p_{e}+\varepsilon \alpha_{0}, \varepsilon \varphi_{0}+d(\varepsilon)\right)$ and denote them by $(v(t, \varepsilon), p(t, \varepsilon), f(t, \varepsilon))$, suppressing all other variables, we obtain, also dividing by $\varepsilon$,

$$
\begin{aligned}
& \left\|\left(\frac{v(t, \varepsilon)-v(0,0)}{\varepsilon}, \frac{p(t, \varepsilon)-p(t, 0)}{\varepsilon}\right)\right\|_{B_{1}\left(\Omega_{t}^{0}\right)}+\left\|\frac{f(t, \varepsilon)-f(t, 0)}{\varepsilon}\right\|_{\widehat{B}_{1}} \\
& \leq C e^{-\gamma t}\left(\left\|\left(\frac{v(0, \varepsilon)-v(0,0)}{\varepsilon}, \frac{p(0, \varepsilon)-p(0,0)}{\varepsilon}\right)\right\|_{B_{1}\left(\Omega_{0}^{0}\right)}+\left\|\frac{f(0, \varepsilon)-f(0,0)}{\varepsilon}\right\|_{\widehat{B}_{1}}\right) .
\end{aligned}
$$

Assuming differentiability as a function of $\varepsilon$ all the quotients stay bounded as $\varepsilon \rightarrow 0$, and so the contribution to the norms coming from the differences between $\Omega_{t}^{0}(\varepsilon)$ and $\Omega_{t}^{0}(0)=\mathcal{R}^{0}$ go to zero as $\varepsilon \rightarrow 0$, assuming also that these norms are obtained by integration of the functions and their derivatives. So letting $\varepsilon \rightarrow 0$ we get

$$
\|(u, \alpha)(t)\|_{B_{1}\left(\mathcal{R}^{0}\right)}+\|\varphi(t)\|_{\widehat{B}_{1}} \leq C e^{-\gamma t}\left(\|(u, \alpha)(0)\|_{B_{1}\left(\mathcal{R}^{0}\right)}+\|\varphi(0)\|_{\widehat{B}_{1}}\right) .
$$


In addition $u(0)=u_{0}, \alpha(0)=\alpha_{0}, \varphi(0)=\varphi_{0}+d^{\prime}(0)=\varphi_{0}$. Due to the density of the " $B_{2}$ spaces" in the " $B_{1}$ spaces" we can now conclude that the linearized equation should exhibit uniform exponential decay, and as we have shown in Theorem 2 this is not the case, so the assumption about exponential decay of the solutions of the non-linear equation also cannot be true. For incompressible fluids we must omit $p$ from consideration and do not need to worry about changing the mass, as then

$$
\int_{0}^{l_{1}} \int_{0}^{l_{2}} \varphi_{0}\left(x_{1}, x_{2}, t\right) d x_{2} d x_{1}=0,
$$

which already assures the mass is constant.

\section{References}

[1] S. Agmon, On the eigenfunctions and on the eigenvalues of general elliptic boundary value problems, Comm. Pure Appl. Math. 15 (1962), 119-147.

[2] S. Agmon, A. Douglis, L. Nirenberg, Estimates near the boundary for solutions of elliptic partial differential equations satisfying general boundary conditions II, Comm. Pure Appl. Math. 17 (1964), 35-92.

[3] J. T. BEALE, The initial value problem for the Navier-Stokes equation with a free surface, Comm. Pure Appl. Math. 34 (1981), 359-392.

[4] A. Friedman, Partial Differential Equations, Holt, Rinehart, Winston, New York, 1969.

[5] P. SECChI, A. VAlli, A free boundary value problem for compressible viscous fluids, J. Reine Angew. Math. 341 (1983), 1-31.

[6] V. A. Solonnikov, On an unsteady motion of an isolated volume of a viscous incompressible fluid, Izv. Akad. Nauk. SSSR Ser. Mat. 51 (1987), 1065-1087 (in Russian); English transl.: Math. USSR-Izv. 31 (1988), 381-405.

[7] G. Ströhmer, About the resolvent of an operator from fluid dynamics, Math. Z. 194 (1987), 183-191.

[8] G. Ströhmer, About the linearized stability of the spherically symmetric solution of the equations of a barotropic viscous fluid under the influence of self-gravitation, preprint.

[9] G. Ströhmer, W. ZajączKowski, On the existence and properties of the rotationally symmetric equilibrium states of compressible barotropic self-gravitating fluids, Indiana Univ. Math. J. 46 (1997), 1181-1220.

[10] H. Triebel, Interpolation Theory, Function Spaces, Differential Operators, North Holland, Amsterdam, 1978.

[11] J. T. Wloka, B. Rowley, B. Lawruk, Boundary Value Problems for Elliptic Systems, Cambridge Univ. Press, Cambridge, 1995.

[12] W. ZAJĄCZKOWski, On nonstationary motion of a compressible barotropic viscous fluid bounded by a free surface, Dissertationes Math. (Rozprawy Mat.) 324 (1993), 101 pp. 